Accelerator Division

Alternating Gradient Synchrotron Department

BROOKHAVEN NATIONAL LABORATORY

Upton, New York 11973

Accelerator Division

Technical Note

AGS/AD/Tech. Note No. 459

AGS PUE Geometry Factor and Normalization

L. Ahrens

March 5, 1997 


\title{
AGS PUE Geometry Factor and Normalization
}

\author{
L. Ahrens
}

\section{Introduction}

The subject of this note is the connection between the amplitude of beam induced electrical signals obtained from the AGS "conversion" pick-up electrodes (PUEs) and the position of the beam within those PUEs. In an ideal split cylinder PUE geometry (closely approximated e.g. by the Booster PUEs) with the sum and difference of the two plate signals available, there is a simple linear relation between the beam position and the available signals, namely the position $\mathrm{x}=(\mathrm{k}) *(\mathrm{diff} / \mathrm{sum})$. This $\mathrm{k}$ is the geometric factor which in this ideal geometry is just the radius of the PUE cylinder.

In the AGS the situation is more complicated for two reasons. The PUE plate geometry provides both vertical and horizontal position information from two pairs of plates which physically occupy a longitudinal distance even shorter than the distance used by the Booster PUEs. The geometry is not a simple split cylinder. Rather the cylinder is cut into four curved "triangular" pieces, the horizontal triangles split vertically and complete the cylinder at one end, and then taper to points lying in the horizontal center plane on the two sides. The vertical plates do the same though rotated by $90^{\circ}$ starting from the other end of the cylinder, and from a horizontal split. The two pairs fit closely together. The question of what is to be used for " $\mathrm{k}$ ", and what is to be used for the "sum" in extracting vertical and horizontal position information must be specified.

A second complication in AGS is that the sum information for individual PUEs is in fact not available. Rather an independent signal proportional to the average beam intensity is used to do the normalization in the Equilibrium Orbit Acquisition system. This fact has reduced the importance of knowing the $\mathrm{k}$ for the geometry since an additional multiplicative factor is necessarily introduced from the intensity measurement. Only the product of the two is relevant for (or can be learned from) the final position result. However signals from the individual plates of AGS PUEs are available, and are used e.g. for measuring beam coherence. For this sort of work a knowledge of the geometry factor is very useful.

It is a standard technique at AGS to extract the calibration for the Orbit system by scanning the beam momentum, on a magnetic porch - a region in the acceleration cycle where the main magnet field is not changing - and recording the change in the rf frequency and the change in the reported beam position across the scan. The if frequency change implies a change in the beam momentum; if the dispersion at the PUE is known, this then implies a specific (horizontal) position shift at the PUE. Typically this is done for the ring as a whole, averaging the reported motion from all of the PUEs which are nominally all located at points of average dispersion. The high level code is then adjusted to make the reported motion in the horizontal plane agree with the predicted motion. The vertical PUE calibration is set equal to the horizontal. Although this procedure satisfies the immediate needs from the Orbit program, it doesn't yield a number for $\mathrm{g}$. 
During the FY97 gold run, the G14 PUE signals were available in the MCR. An attempt was made to extract the geometry factor using the basic momentum scan technique described in the last paragraph while looking directly at the signals from the two horizontal plates. In analyzing that data, it became clear that a knowledge of the geometry factor from a bench measurement would be valuable, given that the dispersion function at a particular PUE is not an independently measured number. Such a bench measurement was possible given that a very realistic duplication of a (ring magnet/vacuum chamber with PUE) existed in the AGS assembly area. A wire was run through that vacuum chamber and a low frequency response measurement made. That measure is described.

\section{Measurement Setup}

A reasonably thin wire (about .01 inch diameter) is deployed through the vacuum chamber. The wire is mildly tensioned to form a smooth nearly straight line from end to end. The "can" containing the PUE included a bellows section to the outside, making the boundary conditions at the ends of the PUE realistic. The far end of the wire and the vacuum chamber were connected across the output from a signal generator which produced a somewhat smoother "square wave" voltage wave form with amplitude 10 Volts and period 2 microseconds. For a centered wire, each of the four plates of the PUE responded with an identical voltage wave form but with an amplitude of about 5 $\mathrm{mV}$. The induced voltage amplitudes on the four plates were simultaneously averaged, ultimately for over 1000 cycles; and recorded. Four channel digital scopes are wonderful things. The position of the wire, at the PUE end of the vacuum chamber was then systematically moved, first in the horizontal and then in the vertical planes, with the 1000 cycle measurement repeated at each position. The data was taken in two sessions. The first involved the horizontal plane only, with relatively crude $(1 \mathrm{~mm})$ wire placement precision but a large range of motion. The second session explored both planes, with higher precision but a more limited range of motion. Graphs of the data are presented in Figures 1 through 4.

The results are informative in several respects. The slopes of the normalized difference vs wire position lines should yield the desired geometric factor. A choice for normalization must be made, and the data allow three relevant options. One can pretend that the two plates in one plane form a split cylinder and simply divide the difference by the sum in that plane. This approach results in the " 2 plate" sum data in the figures. Alternatively one can normalize by the sum over all four plates. Finally, one can simply normalize for this set of data by a constant, i.e. divide the difference between the plates by a constant. This last approach is equivalent to the normalization actually used in AGS since the amplitude of the signal on the wire was not changed during the data taking. The three approaches give the three behaviors seen in Figures 1 and 2 . A deviation from linearity is obvious in all three cases. The "2 sum" is worse than the "constant" which is worse than the "4 sum". The straight line shown in Figure 1 is a fit to the 4 sum data over only the four central points. The constant used in the "constant" normalization case is adjusted to make the data treated this way agree with the 4 sum line, again for the central four points. To aid in the interpretation, the vertical scale in Figure 2 is put into millimeter units using the slope from the straight line fit. For $+/-1 \mathrm{~cm}$ motion at the PUE origin the three normalizations yield the correct position to better than a mm. The 4 sum and constant normalization deviate from truth by $+/-1 \mathrm{~mm}$ at about $+/-3 \mathrm{~cm}$ from the PUE center. This is a very large beam excursion under normal conditions. There are special times when 
it is exceeded e.g. at the peak of the extraction bump moving the beam into the G10 kicker but they are rare.

The data in Figure 2 suggests that the slope might be steeper right at the origin. To look again and closer at this, and to investigate the vertical response, the wire was attached to the traversing stage from a microscope mount, and the experiment repeated. (The sense of the horizontal motion was inadvertently reversed in this data set, and has been left in that state.) The data in Figures 3 and 4 resulted. For Figure 3 the horizontal scale is now millimeters instead of centimeters. The reproducibility and quality of the fit to a straight line suggests the system was reproducing at the .1 $\mathrm{mm}$ level. The slope extracted from this full set agrees to within $2 \%$ with the slope from the central four points of Figure 1.

Because of the limited range of the wire mover, the vertical data was taken with two setups. The sets are combined in Figure 4 by shifting the vertical position for one of the sets such that the point with the same electrical output in the two sets overlay in position. Then the full set is refit to extract a vertical geometry slope.

In order to derive the best estimate for the geometry factor from this data one other trivial fact has to be corrected for. The measured wire motion which occurs at the end of the setup geometry is slightly greater than the motion at the PUE itself. The wire at the far end of the chamber is left fixed. An estimate for this effect is obtained by measuring the relative lengths involved, and approximating the effective position for the horizontal and vertical plates relative to the wire end points. This correction required the naively extracted horizontal geometry factor be reduced by .905 and the vertical by .873 so this is a $10 \%$ correction. As a check both ends of the wire were moved together. The motion reported by the PUE is consistent with this correction, but the precision of the far end movement is not adequate to refine the estimate. The extracted geometry factors, at the PUE center are then: $40 \mathrm{~mm}$ in the horizontal and $46 \mathrm{~mm}$ in the vertical. The beam position is this factor multiplied by twice the difference divided by the sum of all four plates, or just the difference divided by the sum of the two plates in the same plane.

\section{Comments on these Values}

Although there must be ancient (these PUEs were installed into the AGS ring first in about 1970) evaluations of this constant, D. Ciardullo has relatively recently measured the factor for an oversized PUE identical to the one at $\mathrm{H} 8$ using a bench jig. He reported a geometric factor of $47 \mathrm{~mm}$, apparently measured in the spirit of a two plate normalization. The PUE was radially oversized relative to a normal unit by $(163 \mathrm{~mm} / 151 \mathrm{~mm})$. Scaling the geometry factor by this ratio would reduce his result for a normal PUE to $43.5 \mathrm{~mm}$. So this determination falls just between the two results from the above AGS measurement. Indeed is there any reason to expect the two planes to have different geometry factors? There is at least one possibly relevant lack of symmetry, namely the AGS vacuum chamber itself, which closes in vertically very sharply both upstream and downstream of the cylindrical PUE can while the horizontal chamber stays at the PUE can radius. This could introduce different (beam to plate) capacitance and hence different geometry factors for the two planes - which incidentally should be carried through even in the present AGS Equilibrium Orbit system. A measurement using another AGS chamber would be a satisfying confirmation of this possibility. 
The center line of the PUE can is physically mounted slightly away from the ideal beam orbit in the horizontal plane. This offset is different for different PUEs but on average puts the ideal beam inside of the PUE center by about $10 \mathrm{~mm}$. The data in Figures 1 and 2 suggest why the builders of the PUE system originally attempted to move the PUE cans closer to the ideal orbit - to maximize the linear region around the ideal beam position. That move has been taken out. It caused more harm than good; but as a consequence the deviation from linearity of the system is increased for a beam located inside of the ideal orbit. The effect becomes measurable for about a two centimeters inward shift.

Returning finally to the G14 PUE momentum scan which motivated this measurement, the data is shown in Figure 5. The variation, difference/sum is a reasonably linear function of the change in $\mathrm{rf}$ frequency. When this is translated into a geometry factor, assuming the G14 dispersion is the ring average $\left(D_{x}=1.78 \mathrm{M}\right)$, a value of $39.8 \mathrm{~mm}$ results. (This result is obtained after compensating for an after the fact measurement of the relative gains on the two plates which found that they differed by $20 \%$ and which pulled the geometry factor down to 38.6). Alternatively, the dispersion for G14 (or any \#14 straight section) can be used in the extraction of the geometry factor. For the $15 \mathrm{GeV} / \mathrm{c}$ "bare" machine the G14 dispersion is predicted to be $\mathrm{D}_{\mathrm{x}}=1.67 \mathrm{M}$ and the extracted geometry factor becomes $37.3 \mathrm{~mm}$. A prediction for the dispersion for the machine as configured during the study could be but has not been obtained. In retrospect the results are in reasonable agreement; a priori the author had a quite different value in his memory. One additional note, the radius of the effective cylinder that the PUE plates make up is 6 inches $(O D)$ with the plates themselves $1 / 16$ inch thick, hence the inner radius of this geometry is $(3-1 / 32)$ inches or $75.4 \mathrm{~mm}$.

\section{Acknowledgments}

Thanks to Siegfried Naase for help in finding the required pieces, to Barry Clay and the AGS Instrumentation Group for instrumentation, and to Steve Gill and the Vacuum Group for the chamber and wire pulling. 
AGS PUE Geometric Calibration

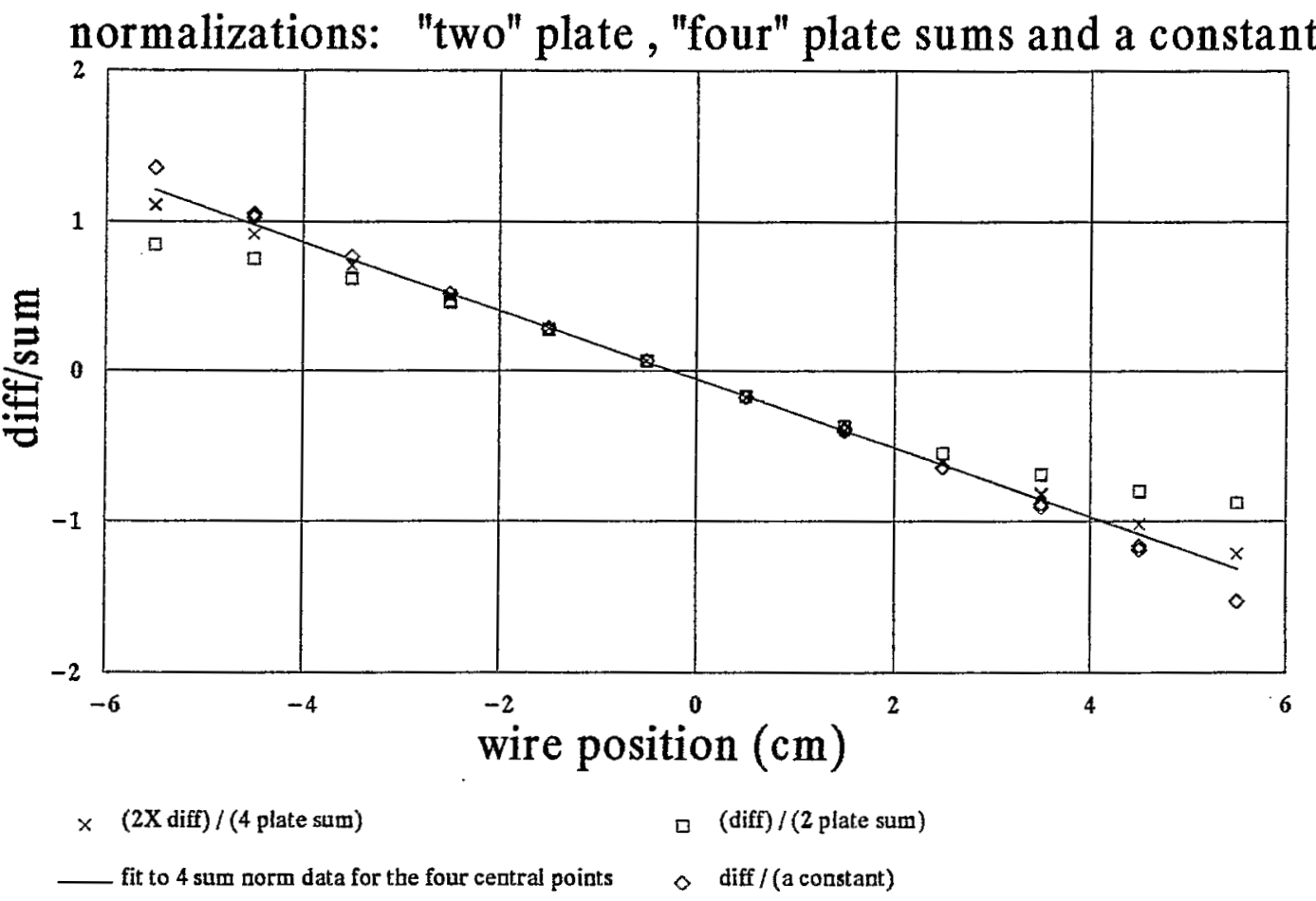

Figure 1.

fit: $-.055-.2292 *$ (position in $\mathrm{cm}$ )

including correction for fized wire at far end [.905], this corresponding to a geometric factor of $39.5 \mathrm{~mm}$

AGS PUE Geometric Calibration

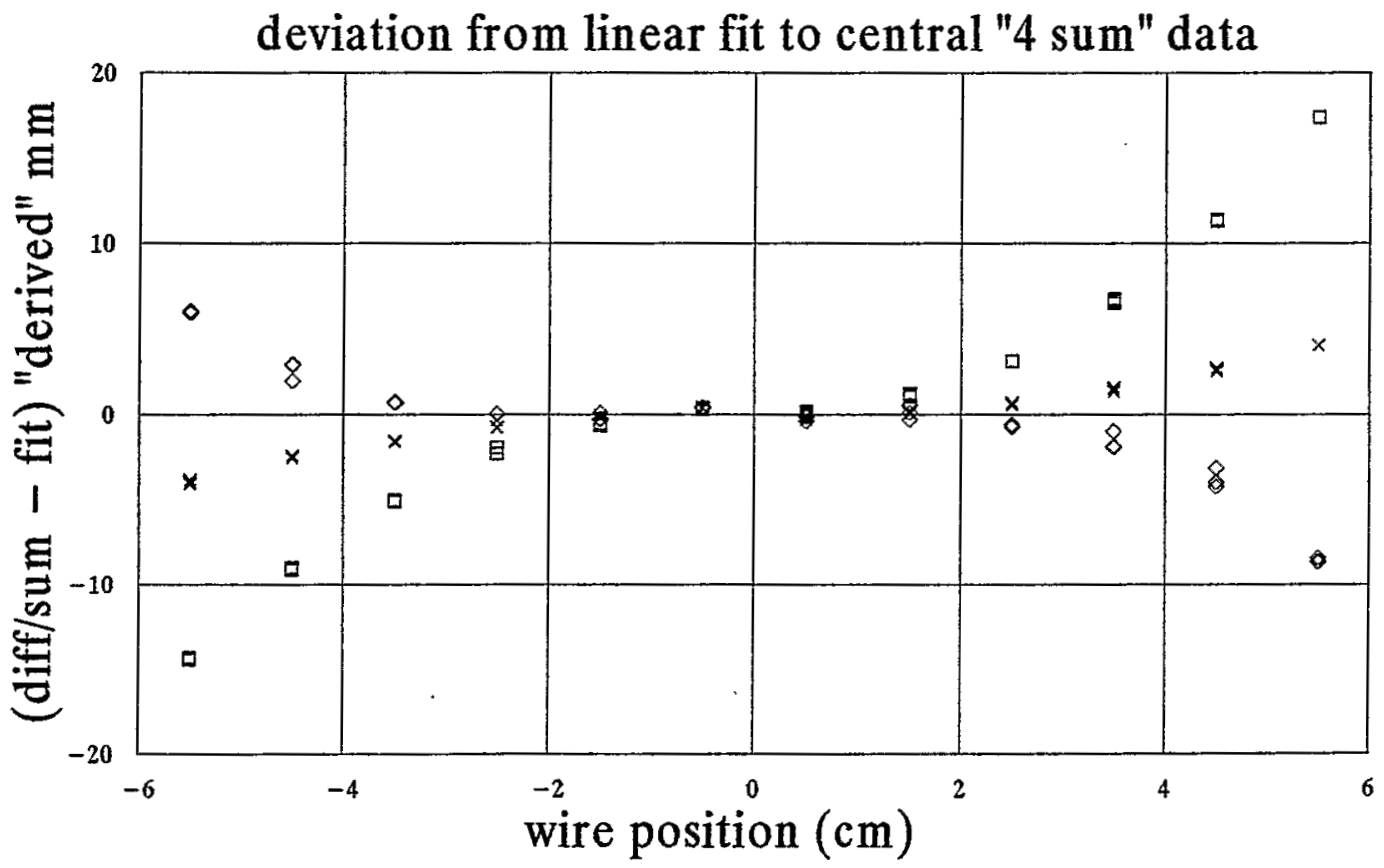

Figure 2.

$\times$ norm to 4 plate sum norm to 2 plate sum

- norm to a constant 
$-6-$

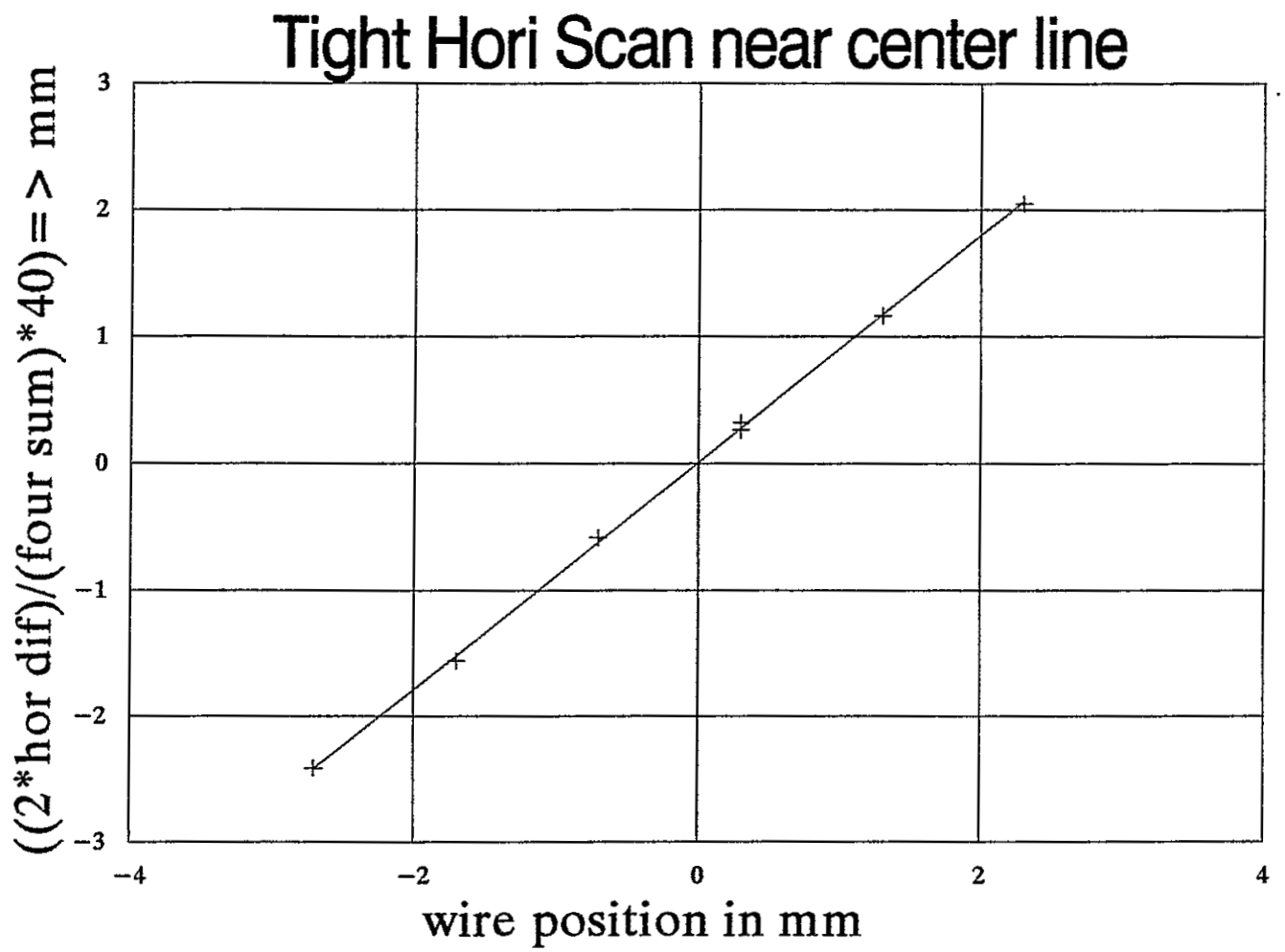

Figure 3 .

+ data _ fit

fit: $.000198+.022418^{*} \mathrm{x}(\mathrm{mm})$; extracted geometry factor (after corr [.905] for fixed wire end) $40.4 \mathrm{~mm}$ data taken 9Feb97, reported wire pos shifted to give 0 at cntr i.e. where diff $=0$

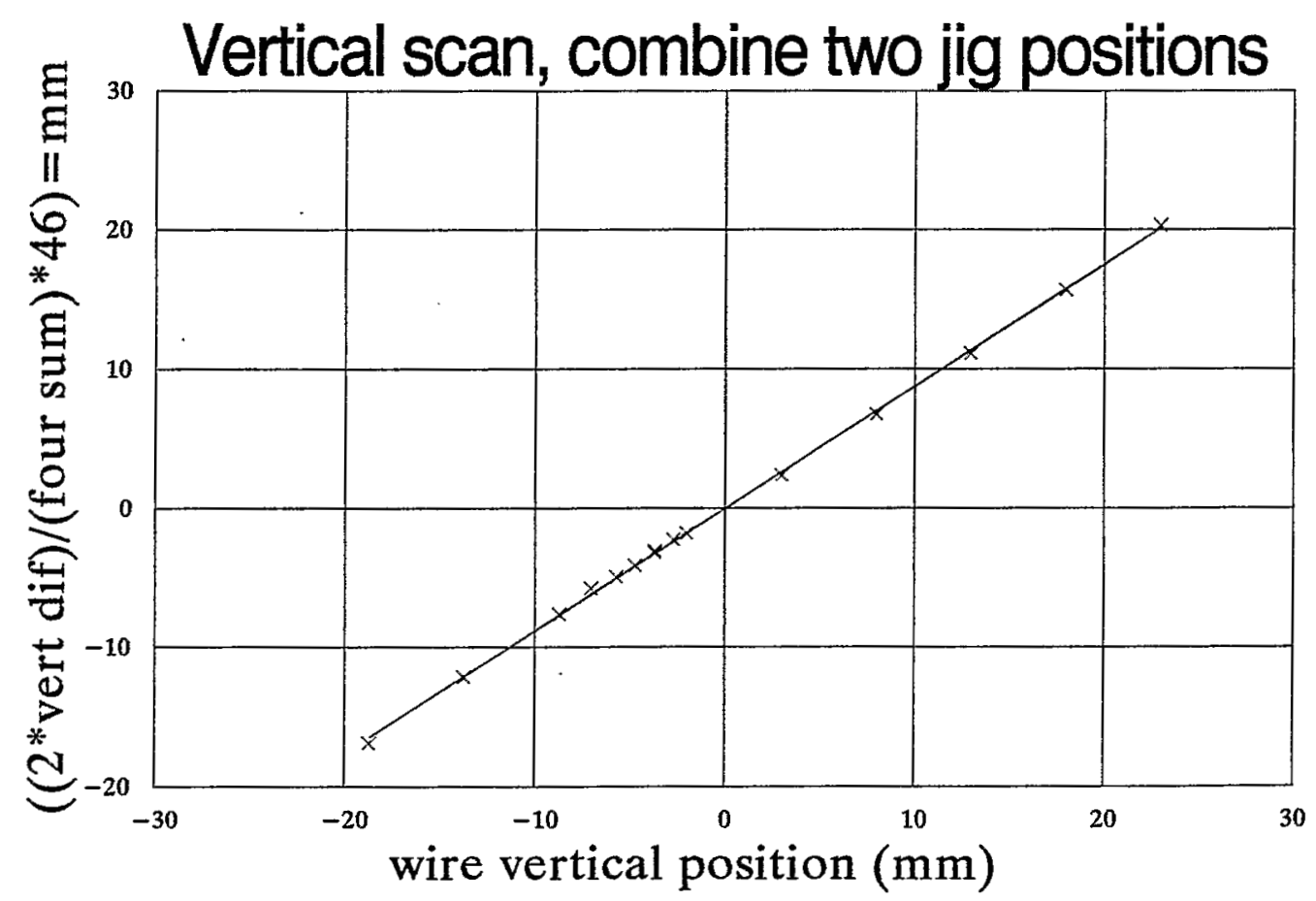

Figure 4.

$\times$ data (both sets combined)

__ fit to both sets, with shift

fit: $-.00078+.01909^{*} \mathrm{y}(\mathrm{mm})$; derived geom factor with corr for fixed wire end $[.873]=46 \mathrm{~mm}$ position for the two data sets globally shifted to give 0 for diff $=0$ 


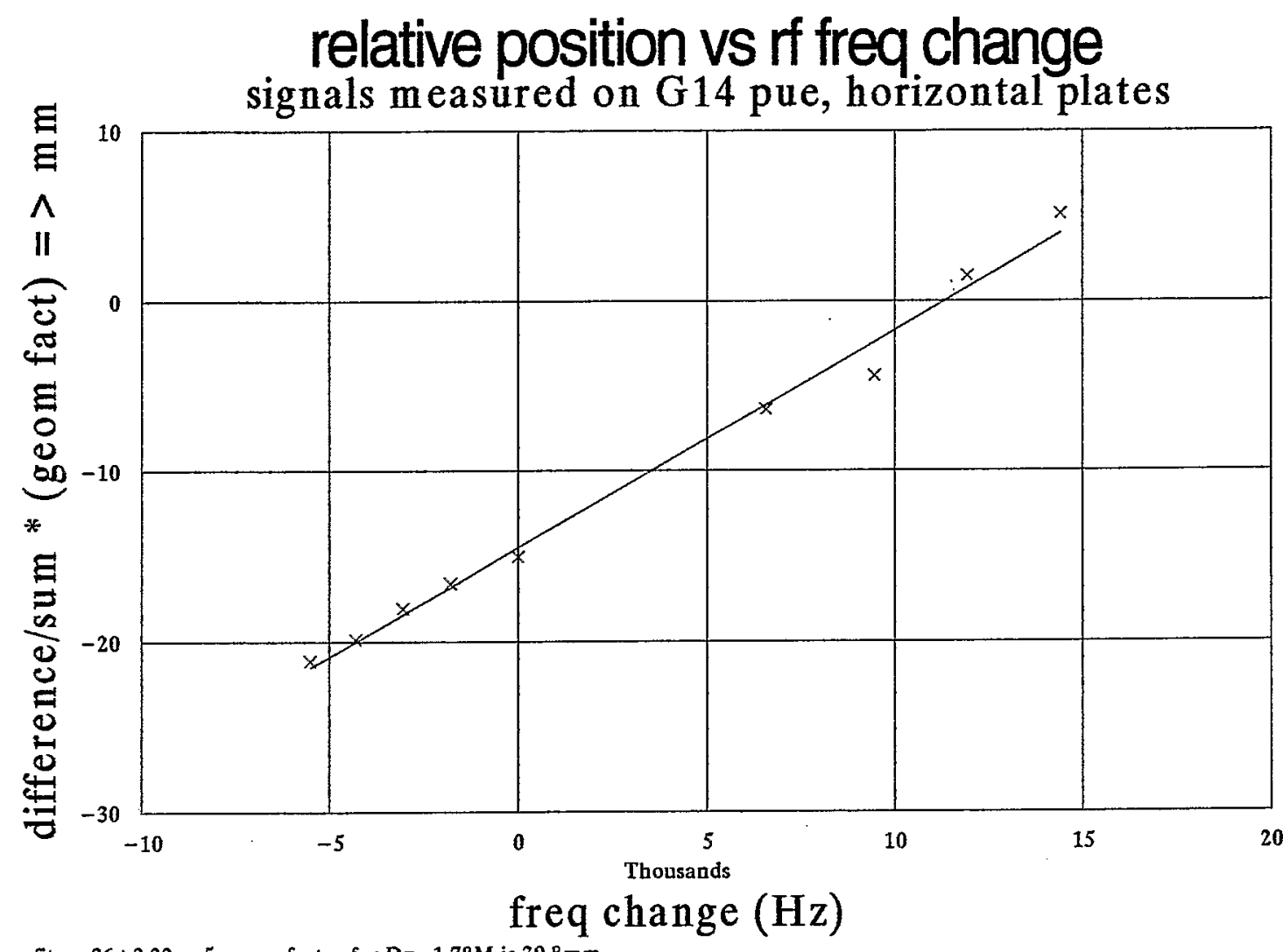

Figure 5.

fit: $-.36+3.22 e-5$, geom factor for $D x=1.78 \mathrm{M}$ is $39.8 \mathrm{~mm}$

here increase measured signals from cable \#3 by $20 \%$ based on post study gain calibration 